\title{
Several types of residuals in cox regression model : an empirical study
}

\begin{abstract}
There are several methods for calculating residual in survival analysis, especially in Cox regression model by which each method has specific use, such as goodness-of-fit, to identify possible outliers and influential observations, or in general to check necessary assumptions. In this article, we study four methods of residuals, namely Schoenfeld, Martingale, deviance, and score residuals and we applied those methods on cardiovascular data.
\end{abstract}

Keyword: Cox regression; Goodness-of-fit; Influential observations; Outliers; Residuals. 